\title{
RELATIONS BETWEEN FUNCTION SPACES
}

\author{
G. G. LORENTZ 1
}

1. Introduction. Let $B$ be a conditionally $\sigma$-complete ring of subsets of a set $S$. We assume that $W(e)$ is a positive function defined for $e \in B$ with the properties:

(1) $W$ is increasing: $W\left(e_{1}\right) \leqq W\left(e_{2}\right)$ if $e_{1} \subset e_{2}$,

(2) $W$ is concave: $W\left(e_{1} \cup e_{2}\right)+W\left(e_{1} \cap e_{2}\right) \leqq W\left(e_{1}\right)+W\left(e_{2}\right), e_{1}, e_{2} \in B$,

(3) $W$ is continuous: $W\left(e_{n}\right) \rightarrow 0$ if $e_{n} \in B$ decreases to 0 .

If $f$ is a $B$-measurable real function defined on $S$, then for each $y>0$ the set $f^{-1}(y)=[x:|f(x)|>y]$ belongs to $B$. The space $\Lambda(W)[3 ; 1]$ consists of all measurable functions $f$ for which

$$
\|f\|=\int_{0}^{+\infty} W\left(f^{-1}(y)\right) d y<+\infty .
$$

With this norm, $\Lambda(W)$ is a Banach space and even a Banach lattice, i.e., a vector lattice with the property that $|g| \leqq f, f \in \Lambda(W)$ for a measurable $g$ implies $g \in \Lambda(W)$ and $\|g\| \leqq\|f\|$. Condition (3) ensures $[1$, Theorem 5$]$ that the norm (4) is continuous with respect to monotone limits.

One obtains examples of such functions $W$ as follows. Let $(S, B, \mu)$ be a measure space with measure $\mu$, which we shall in this paper assume nonatomic. If $\Phi_{0}(u)$ is a positive increasing concave function of $u \geqq 0$ with $\Phi_{0}(0+)=0$, then one sees that $W(e)=\Phi_{0}(\mu e)$ satisfies (1), (2), (3).

An Orlicz space $L_{\Phi}(S)$ is defined by an increasing convex function $\Phi(u), u \geqq 0$ with $\Phi(0+)=0$ and $\Phi^{\prime}(u) \rightarrow \infty$, and by a measure space $(S, B, \mu)$.

We have $f \in L_{\Phi}$ if

$$
\|f\|=\sup \int_{S}|f| g d \mu<+\infty ;
$$

the supremum is taken for all measurable $g \geqq 0$ with $\int_{S} \Psi(g) d \mu \leqq 1$, and $\Psi(u)$ is the conjugate (in the sense of Young) of $\Phi$. This means that $\Phi(u)$ and $\Psi(u)$ are integrals over $(0, u)$ of two monotone positive finite functions $\phi(t), \psi(t)$ which increase to $+\infty$ for $t \rightarrow+\infty$ and are

Received by the editors March 2, 1960 and, in revised form, April 13, 1960.

1 This research was in part supported by the United States Air Force under Contract No. AF 49(638)-619 monitored by the AF Office of Scientific Research. 
inverses of each other. For an exposition of the theory of Orlicz spaces see $[5 ; 2]$.

The problems solved in this note are the following. When is a given space $L_{\Phi}$ also some space $\Lambda(W)$ ? Or when does a given $\Lambda(W)$ coincide with some $L_{\Phi}$ ? Theorems 2 and 3 below show that this can happen only in exceptional cases. We conjecture that this is also true if the $L_{\Phi}$ are replaced by modulared semi-ordered spaces of Nakano [4].

2. Largest space $\Lambda(W)$ contained in $L_{\Phi}$. We start by deriving consequences from $X \subset Y$, where $X$ and $Y$ are Banach lattices of $B$ measurable functions. Let $X$ have the "Fatou property": a measurable function $f$ belongs to $X$ and $\|f\|=\lim \left\|f_{n}\right\|$ if $0 \leqq f_{n}(x) \uparrow f(x)$ and $\sup \left\|f_{n}\right\|<+\infty$. (Thus, spaces $\Lambda\left[1\right.$, Theorem 5] and $L_{\Phi}$ have this property.) Then $X \subset Y$ implies that for some constant $C$,

$$
\left\|\chi_{e}\right\|_{Y} \leqq C\left\|_{\chi_{e}}\right\|_{X} .
$$

Here $\chi_{e}$ is the characteristic function of an arbitrary set $e \in B$. For otherwise $e_{n} \in B$ would exist with $\left\|\chi_{e_{n}}\right\|_{Y}>n^{3}\left\|\chi_{e_{n}}\right\|_{X}$, and for the function $f=\sum_{1}^{\infty} f_{n}, f_{n}=\chi_{e_{n}}\left\|\chi_{e_{n}}\right\|_{\bar{X}}^{-1}$ we would have $f \in X, f \notin Y$.

From this and the definition (4) we obtain for two spaces $\Lambda$, corresponding to the same $(S, B)$ : The inclusion $\Lambda\left(W_{1}\right) \subset \Lambda\left(W_{2}\right)$ is equivalent to $W_{2}(e) \leqq C W_{1}(e), e \in B$, for some constant $C$.

Theorem 1. Let $L_{\Phi}$ be an Orlicz space for the measure space $(S, B, \mu)$. Then there exists a largest space $\Lambda(W)$ of $B$-measurable functions contained in $L_{\Phi}$, namely the space $\Lambda\left(W_{0}\right)$ with

$$
W_{0}(e)=\Phi_{0}(\mu e), \quad \Phi_{0}(x)=x \Psi^{-1}\left(\frac{1}{x}\right), \quad x>0, \quad \Phi_{0}(0)=0 .
$$

Proof. Consider the function

$$
W_{0}(e)=\left\|\chi_{e}\right\|_{L_{\Phi}}=\sup _{\int_{\Psi(o) d \mu=1 ; g \geqq 0}} \int_{e} g(x) d \mu(x) .
$$

Assuming $\mu e>0$, let $g$ be an arbitrary positive measurable function with $\int_{\theta} \Psi(g) d \mu=1$; we define $C$ and $C_{1}$ by

$$
\mu e \Psi(C)=1 \text { and } C_{1}=(\mu e)^{-1} \int_{e} g d \mu .
$$

By Jensen's inequality

$$
\mu e \Psi\left(C_{1}\right) \leqq \int_{e} \Psi(g) d \mu=1,
$$


hence $\Psi\left(C_{1}\right) \leqq \Psi(C)$ and $C_{1} \leqq C$. This means that $\int_{e} C d \mu \geqq \int_{e} g d \mu$, i.e., that the supremum in (8) is attained for $g=C$. Thus we obtain that $W_{0}(e)$ defined by (8) is identical with the function (7). Next we have, if $x \neq 0, y=\Psi^{-1}(1 / x), t=\psi(y)$, and if $\Psi^{\prime}(y)$ exists, i.e., the function $\psi$ is continuous at $y$,

$$
\begin{aligned}
\Phi_{0}^{\prime}(x) & =\Psi^{-1}\left(\frac{1}{x}\right)-\frac{1}{x \Psi^{\prime}(y)} \\
& =y-\frac{\Psi(y)}{\Psi^{\prime}(y)} \\
& =\frac{\Phi(t)}{t},
\end{aligned}
$$

because in this case $\Psi(y)+\Phi(t)=y t$.

If $\psi$ is discontinuous at $y$, then still the right and the left derivatives of $\Phi_{0}$ at $x$ are equal to $t^{-1} \Phi(t)$ with $t=\psi(y-)$ or $t=\psi(y+)$, respectively. This shows that $\Phi_{0}(x)$ has positive decreasing derivatives, hence $\Phi_{0}(x)$ is an increasing concave function. Since $y^{-1} \Psi(y)$ $\rightarrow \infty$ for $y \rightarrow \infty$, we have $\Phi_{0}(0+)=0$. Thus the function (7) satisfies (1), (2), (3).

Considering $Y=\Lambda\left(W_{0}\right)$, we see that $\|f\|_{X} \geqq\|f\|_{L_{\Phi}}$ holds [1, Theorems $1,2]$ for each step function $f$. The same relation for an arbitrary $f \geqq 0$ in $Y$ follows from the Fatou property of $L_{\Phi}$ : from (4) one derives that $f$ is the limit of an increasing sequence of positive step functions $f_{n}$ for which $\lim \sup \left\|f_{n}\right\|_{L_{\Phi} \leqq} \lim \left\|f_{n}\right\|_{Y}=\|f\|_{Y}$. Thus $f \in L_{\Phi}$ and $Y \subset L_{\Phi}$. Finally, any other $\Lambda$-space contained in $L_{\Phi}$ is also contained in $\Lambda\left(W_{0}\right)$. For $\Lambda(W) \subset L_{\Phi}$ implies by (6) $W_{0}(e) \leqq C W(e)$ and $\Lambda(W)$ $\subset \Lambda\left(W_{0}\right)$.

3. Conditions for the equality of the spaces $\Lambda, L_{\Phi}$. From (4) one derives that each function $f \in \Lambda(W)$ can be approximated in the $\Lambda(W)$-norm by step functions. The spaces $L_{\Phi}$ do not possess in general this property. Let $l=\mu S$; this approximation is possible if and only if constants $M$ and $u_{0}>0$ exist with

$$
\Phi(2 u) \leqq M \Phi(u) \text { for } \begin{cases}u \geqq u_{0} & \text { if } l<+\infty \\ u>0 & \text { if } l=+\infty\end{cases}
$$

Sufficiency of this condition is known [5, p. 84]. We shall prove its necessity for $l<+\infty$ (the other case is treated in a similar fashion).

If (10) were not true, we could construct by induction sequences $u_{n} \rightarrow \infty$ and $\delta_{n}>0$ with $\sum_{k=1}^{n} \delta_{k}<l$ as follows. At the $n$th step, we take $u_{n}>n$ so that $\Phi\left(2 u_{n}\right)>2^{n} \Phi\left(u_{n}\right)$. By the induction hypothesis, $\sum_{1}^{n-1} \delta_{k}$ 
$<l$. We can assume $u_{n}$ so large that $\sum_{1}^{n} \delta_{k}<l$ with $\delta_{n}=2^{-n} \Phi\left(u_{n}\right)^{-1}$. Since $\mu$ is nonatomic and therefore full-valued, we can find disjoint measurable sets $e_{n} \in B$ with $\mu e_{n}=\delta_{n}$. Put $f(x)=3 u_{n}$ for $x \in e_{n}, n=1,2$, $\cdots, f(x)=0$ for all other points of $S$. Then $f \in L_{\Phi}$, since (see [5, p. 79])

$$
\int_{S} \Phi\left(\frac{1}{3} f\right) d \mu=\sum_{n=1}^{\infty} \Phi\left(u_{n}\right) \delta_{n}<+\infty .
$$

On the other hand, if $f_{1}$ takes only a finite number of values, then $f_{1}(x)$ is bounded, hence $f(x)-f_{1}(x) \geqq 2 u_{n}$ for $x \in e_{n}$ and all large $n$. Therefore

$$
\int_{S} \Phi\left(\left|f-f_{1}\right|\right) d \mu \geqq \int_{e_{n}} \Phi\left(2 u_{n}\right) d \mu=\delta_{n} \Phi\left(2 u_{n}\right)>1 .
$$

But this implies [5, p. 80, Theorem 2] that $\left\|f-f_{1}\right\|_{L_{\Phi}} \geqq 1$, and proves our assertion.

We can now prove our main result:

Theorem 2. Let $L_{\Phi}$ be an Orlicz space for the measure space $(S, B, \mu)$ and let $l=\mu S$. Then $L_{\Phi}$ is equal to a space $\Lambda(W)$ if and only if $\Phi$ satisfies for some $\delta>0$

$$
\int_{\Psi^{-1}\left(l^{-1}\right)}^{\infty} \frac{\Psi(\delta x)}{\Psi(x)^{2}} d \Psi(x)<+\infty .
$$

Proof. First we assume that $\Phi(u)$ satisfies (10). In view of Theorem 1 we have to prove the following. If $W_{0}(e)$ is defined by (7), then $\Lambda\left(W_{0}\right) \supset L_{\Phi}$ is true if and only if (11) is satisfied.

This inclusion $\Lambda\left(W_{0}\right) \supset L_{\Phi}$ means that each $f \in L_{\Phi}, f(x) \geqq 0$, satisfies $\|f\|_{\Lambda\left(W_{0}\right)}<+\infty$ or equivalently

$$
\begin{aligned}
& \int_{0}^{\infty} W\left(f^{-1}(y)\right) d y=\int_{0}^{\infty} \Phi_{0}\left(\mu f^{-1}(y)\right) d y \\
& =\int_{0}^{\infty} d y \int_{0}^{\mu f^{-1}(y)} \Phi_{0}^{\prime}(u) d u=\int_{0}^{l} f^{*}(u) \Phi_{0}^{\prime}(u) d u<+\infty .
\end{aligned}
$$

Here $f^{*}(u)$ denotes the decreasing rearrangement of $f(x)$, that is a decreasing positive function defined on $0<u<l$, equimeasurable with $f(x)$ with respect to the Lebesgue measure on $(0, l)$. Because the measure $\mu$ on $S$ is full-valued, for each $f^{*}$ on $(0, l)$ we can find an equimeasurable positive function $f$ on $S$. Hence, if $f$ runs through all positive functions of $L_{\Phi}(S), f^{*}$ will run through all decreasing $f^{*} \in L_{\Phi}(0, l)$. Since $\Phi_{0}^{\prime}(u)$ is decreasing, (12) is equivalent to $\int_{0}^{l} f \Phi_{0}^{\prime} d u$ 
$<+\infty$ for all positive $f \in L_{\Phi}(0, l)$. But $f \in L_{\Phi}(0, l)$ is equivalent [4, p. 80] with $\int_{0}^{l} \Phi(\delta f) d u<+\infty$ for some $\delta>0$. Thus condition (12) becomes $\int_{0}^{l} f \Phi_{0}^{\prime} d u<+\infty$ for all $f \geqq 0$ with $\int_{0}^{l} \Phi(f) d u<+\infty$ or [4, p. 138] simply $\Phi_{0} \in L_{\Psi}^{0}(0, l)$. Using again (9), we find with $y=\Psi^{-1}(1 / u)$, $t=\Psi^{\prime}(y), y=\phi(t)$,

$$
\begin{aligned}
\Phi_{0}^{\prime}(x) & =\frac{\Phi(t)}{t} \geqq \frac{1}{M} \frac{\Phi(2 t)}{t} \geqq \frac{1}{M t} \int_{t}^{2 t} \phi(u) d u \\
& \geqq \frac{\phi(t)}{M}=\frac{1}{M} \Psi^{-1}\left(\frac{1}{u}\right) .
\end{aligned}
$$

Hence $\Phi_{0}^{\prime} \in L_{\Psi}(0, l)$ is equivalent to

$$
\int_{0}^{l} \Psi\left(\delta \Psi^{-1}\left(\frac{1}{u}\right)\right) d u<+\infty \quad \text { for some } \delta>0 .
$$

Making the substitution $x=\Psi^{-1}\left(u^{-1}\right)$ we see that this is equivalent to (11).

We complete the proof by showing that (11) implies (10). Assume that (10) does not hold. Let for example $\Phi(2 u) / \Phi(u)$ be unbounded for $u \rightarrow \infty$. Then also $\phi(2 u) / \phi(u)$ is unbounded, for an integration of $\phi(2 u) \leqq M \phi(u)$ would give $\Phi(2 u) / \Phi(u) \leqq M_{1}$ for large $u$. There exists therefore a sequence $u_{n}$ with

$$
\phi\left(2 u_{n}\right) \geqq n \phi\left(u_{n}\right), \quad \quad u_{n} \rightarrow \infty .
$$

Putting $v_{n}=\phi\left(u_{n}\right), v_{n}^{\prime}=\phi\left(2 u_{n}\right)$, we find $v_{n}^{\prime} \geqq n v_{n}$ and $\psi\left(v_{n}^{\prime}\right)=2 \psi\left(v_{n}\right)$. Let $\delta>0$ be chosen, then for $v_{n}^{\prime} / 2 \leqq v \leqq v_{n}^{\prime}$ and all large $n$,

$$
\begin{aligned}
\Psi(\delta v) & \geqq \Psi\left(\frac{1}{2} \delta v_{n}^{\prime}\right) \geqq\left(\frac{1}{2} \delta v_{n}^{\prime}-v_{n}\right) \psi\left(v_{n}\right) \\
& \geqq \frac{1}{3} \delta v_{n}^{\prime} \psi\left(v_{n}\right)=\frac{1}{6} \delta v_{n}^{\prime} \psi\left(v_{n}^{\prime}\right) \\
& \geqq \frac{1}{6} \delta \Psi(v) .
\end{aligned}
$$

It is clear that $\Psi\left(v_{n}^{\prime}\right) \geqq 2 \Psi\left(v_{n}^{\prime} / 2\right)$. Hence

$$
\int_{v_{n^{\prime}} / 2}^{v_{n^{\prime}}} \frac{\Psi(\delta u)}{\Psi(u)^{2}} d \Psi(u) \geqq \frac{1}{6} \delta \log \frac{\Psi\left(v_{n}^{\prime}\right)}{\Psi\left(\frac{1}{2} v_{n}^{\prime}\right)} \geqq \frac{1}{6} \delta \log 2 .
$$

This shows that (11) is violated for all $\delta>0$. The case when (10) is 
not satisfied for $u \rightarrow 0$ is handled in the same way. The proof is complete.

There is also a theorem in the opposite direction.

Theorem 3. Let $\Lambda(W)$ with $W(e)=\Phi_{0}(\mu e)$ correspond to a measure space $(S, B, \mu)$, and let $\Phi_{0}(u)$ be an increasing continuous concave function for $u \geqq 0$ with $\Phi_{0}(0)=0$. Let $\Phi_{0}(u)=u P(u), u>0$. Then $\Lambda(W)$ is a space $L_{\Phi}$ if and only if

$$
\int_{0}^{l} \frac{d x}{P^{-1}(\delta P(x))}<+\infty, \quad \text { for some } \delta>0 .
$$

This follows easily from Theorem 2; we leave the details to the reader.

Summing up Theorems 2 and 3 we see that the relation $L_{\Phi}=\Lambda(W)$ holds only in very exceptional cases. The integral (11) diverges for $\delta=1$; hence condition (11) means that $\Psi$ is very rapidly increasing. Thus $\Psi(u)=e^{\log ^{p} u}, p>1$ and $\Psi(u)=e^{\log u \log \log u}=u^{\log \log u}$ satisfy (11). For the space $L^{1}, \Phi(u)=1$ for $u>0$, and $\Psi(v)=\infty$ for $v>1$. We can say that the relation $L_{\Phi}=\Lambda(W)$ can hold only if these spaces are fairly close to $L^{1}$.

\section{REFERENCES}

1. B. J. Eisenstadt, and G. G. Lorentz, Boolean rings and Banach lattices, Illinois J. Math. vol. 3 (1959) pp. 524-531.

2. M. A. Krasnosel'skiY and Ya. B. RutickiY, Convex functions and Orlicz spaces, Moscow, Gosudarstv. Izdat. Fiz.-Mat. Lit., 1958. 37-55.

3. G. G. Lorentz, Some new functional spaces, Ann. of Math. vol. 51 (1950) pp.

4. H. Nakano, Modulared semi ordered linear spaces, Tokyo, Mauruzen Co., 1950.

5. A. C. Zaanen, Linear analysis, New York, Interscience, 1953.

SYRACUSE UNIVERSITY 